\title{
Dark Matter from Exponential Growth
}

\author{
Torsten Bringmann $\odot,{ }^{1, *}$ Paul Frederik Depta $\odot,{ }^{2, \dagger}$ Marco Hufnagel, $3, \$$ \\ Joshua T. Ruderman, ${ }^{4,2,5,6,8}$ and Kai Schmidt-Hoberg $\circledast^{2, \|}$ \\ ${ }^{1}$ Department of Physics, University of Oslo, Box 1048, N-0316 Oslo, Norway \\ ${ }^{2}$ Deutsches Elektronen-Synchrotron DESY, Notkestraße 85, D-22607 Hamburg, Germany \\ ${ }^{3}$ Service de Physique Théorique, Université Libre de Bruxelles, Boulevard du Triomphe, CP225, B-1050 Brussels, Belgium \\ ${ }^{4}$ Center for Cosmology and Particle Physics, Department of Physics, New York University, New York, New York 10003, USA \\ ${ }^{5}$ Kavli Institute for Theoretical Physics, University of California, Santa Barbara, California 93106, USA \\ ${ }^{6}$ School of Physics and Astronomy, Tel-Aviv University, Tel-Aviv 69978, Israel
}

(Received 25 June 2021; revised 20 August 2021; accepted 14 September 2021; published 3 November 2021)

\begin{abstract}
We propose a novel mechanism for the production of dark matter (DM) from a thermal bath based on the idea that DM particles $\chi$ can transform heat bath particles $\psi: \chi \psi \rightarrow \chi \chi$. For a small initial abundance of $\chi$, this leads to an exponential growth of the DM number density in close analogy to other familiar exponential growth processes in nature. We demonstrate that this mechanism complements freeze-in and freeze-out production in a generic way, opening new parameter space to explain the observed DM abundance, and we discuss observational prospects for such scenarios.
\end{abstract}

DOI: 10.1103/PhysRevLett.127.191802

Introduction.-While the identity and underlying properties of the dark matter (DM) in our Universe remain mysterious, its energy density has been precisely inferred by a series of satellite missions studying the cosmic microwave background (CMB). Any theoretical description of DM must therefore include a DM production mechanism that leads to the observed DM relic abundance of $\Omega_{\mathrm{DM}} h^{2} \simeq 0.12$ [1].

A particularly appealing framework for the genesis of $\mathrm{DM}$, minimizing the dependence on initial conditions, is its creation out of a thermal bath. The most commonly adopted paradigm falling into this category is thermal freeze-out from the primordial plasma of standard model (SM) particles in the early Universe [2]. However, given the increasingly strong constraints on this setup, a plethora of alternate production scenarios with DM initially in thermal equilibrium have recently been proposed, including "hidden sector freeze-out" [3-9], "Forbidden DM" [10,11], "Cannibal DM" [12,13], "Coscattering DM" [14-16], "Zombie DM" [17], "Elder DM" [18], "Kinder DM" [19], and "SIMP DM" [20-22]. Another possibility is that DM never entered thermal equilibrium at all, in which case it can be produced via a "leakage" out of a thermal bath, often referred to as "freeze-in" [23,24]. While a large

Published by the American Physical Society under the terms of the Creative Commons Attribution 4.0 International license. Further distribution of this work must maintain attribution to the author(s) and the published article's title, journal citation, and DOI. Funded by SCOAP ${ }^{3}$. number of variants of the freeze-out paradigm have been suggested, less model building has been performed around the freeze-in idea (see, however, Refs. [25-32]).

In this Letter, we propose a novel and generic DM production scenario between these two polarities based on the idea that a DM particle $\chi$ can "transform" a heat bath particle $\psi$ into another $\chi$; cf. Fig. 1. For a small initial abundance $n_{\chi}$, as shown below, this results in an exponential growth of the DM abundance. To be in accord with observations, the exponential growth is required to shut off before the DM particle $\chi$ is fully thermalized, so one can also think of this mechanism as a "failed thermalization." Interestingly, the exponential growth of $n_{\chi}$ comes to an end naturally in our framework, so that the observed DM abundance is readily obtained.

A novel DM production mechanism.-Quantitatively, the evolution of the DM number density $n_{\chi}$ is governed by the Boltzmann equation

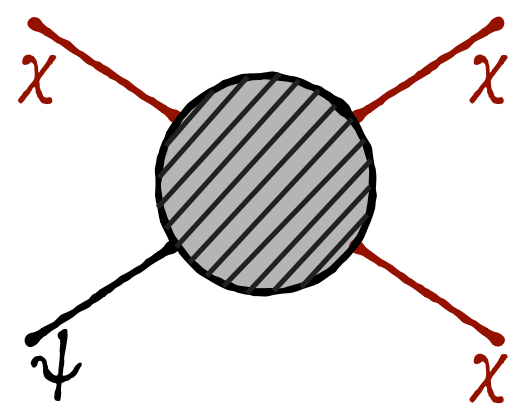

FIG. 1. The transformation process leading to exponential production of DM $(\chi)$ from the heat bath $(\psi)$. 


$$
\dot{n}_{\chi}+3 H n_{\chi}=\langle\sigma v\rangle_{\mathrm{tr}} n_{\chi} n_{\psi}^{\mathrm{eq}}-\langle\sigma v\rangle_{\mathrm{an}} n_{\chi}^{2} .
$$

Here, $n_{\psi}^{\mathrm{eq}}$ is the number density of $\psi$ in equilibrium, $H$ is the Hubble rate, and $\langle\sigma v\rangle_{\mathrm{tr}}\left(\langle\sigma v\rangle_{\mathrm{an}}\right)$ is the cross section for the process $\chi \psi \rightarrow \chi \chi(\chi \chi \rightarrow \chi \psi)$, averaged over the phase space of the initial state. We assume that $\psi$ is in equilibrium with the SM heat bath; this equilibrium can be maintained, for example, by rapid annihilations of $\psi \psi \psi$ to SM states. We note that the zombie collisions of Refs. [17,33] involve a similar process to Fig. 1, but the roles of the DM and bath particles are reversed.

As long as $n_{\chi}^{\mathrm{eq}} \gg n_{\chi}$, we can neglect the second term on the rhs of the above equation. Introducing $x_{\psi} \equiv m_{\psi} / T$ and $Y_{\chi} \equiv n_{\chi} / s$, with $s$ being the entropy density of the heat bath, the solution of the Boltzmann equation is given by

$$
Y_{\chi}\left(x_{\psi}\right) \simeq Y_{\chi}^{0} \exp \left[\int_{x_{\psi}^{0}}^{x_{\psi}} \frac{d x}{x} P(x)\right] \text {, }
$$

where

$$
P(x)=\tilde{H}^{-1} n_{\psi}^{\mathrm{eq}}\langle\sigma v\rangle_{\mathrm{tr}} .
$$

Here, $Y_{\chi}^{0}$ denotes the DM abundance at some initial "time" $x_{\psi}^{0}$, and we have defined $\tilde{H} \equiv H /\left[1+(1 / 3) d\left(\log g_{\text {eff }}^{s}\right) /\right.$ $d(\log T)]$, where $g_{\text {eff }}^{s}$ encodes the entropy degrees of freedom.

Equation (2) describes exponential growth of the DM abundance, with growth rate $P$, as long as $P^{\prime}(x)>0$. For highly relativistic heat bath particles (with $n_{\psi}^{\mathrm{eq}} \propto x_{\psi}^{-3}$ ), this is automatically achieved for $\langle\sigma v\rangle_{\mathrm{tr}}=(\sigma v)_{\mathrm{tr}}^{0} x_{\psi}^{k}$, with $(\sigma v)_{\mathrm{tr}}^{0} \simeq$ const. and $k>1$, i.e., infrared (IR) dominated transformation processes since $H \propto x_{\psi}^{-2}$. Later, once the heat bath particles become nonrelativistic, exponential growth will inevitably come to an end for any value of $k$ due to the Boltzmann suppression of $n_{\psi}^{\text {eq }}$, leading to an asymptotically flat $Y_{\chi}\left(x_{\psi}\right)$. Parametrically, we thus find

$$
Y_{\chi}^{\mathrm{final}} \sim Y_{\chi}^{0} \exp \left[\frac{\lambda_{*}}{k-1} x_{\psi, \mathrm{NR}}^{k-1}\right]
$$

for the final DM abundance, where $x_{\psi, \mathrm{NR}} \sim 3$ and $\lambda_{*} \sim 6 \times 10^{-2} g_{\psi} g_{\mathrm{eff}}^{-\frac{1}{2}} m_{\psi} m_{\mathrm{Pl}}(\sigma v)_{\text {tr }}^{0}$, with $g_{\mathrm{eff}}$ the energy degrees of freedom and $m_{\mathrm{PI}}$ the Planck mass.

We confirm this expectation in Fig. 2, where we show (with solid lines) the full solution of Eq. (1), adopting for illustration a constant amplitude $\left|\mathcal{M}_{\mathrm{tr}}\right|^{2}=\lambda_{\mathrm{tr}}^{2}$, which is realized if $\chi$ and $\psi$ are real scalars with interaction $\mathcal{L} \supset\left(\lambda_{\text {tr }} / 3 !\right) \psi \chi^{3}$. We calculate, for simplicity, $\langle\sigma v\rangle_{\text {tr }}$ for a Maxwell-Boltzmann distribution [34], leaving a determination of its precise phase space distribution for future work; this leads to $\langle\sigma v\rangle_{\mathrm{tr}} \propto T^{-2}$ for $T \gg m_{\chi}, m_{\psi}$. Starting from an initial value of the DM abundance (indicated by the

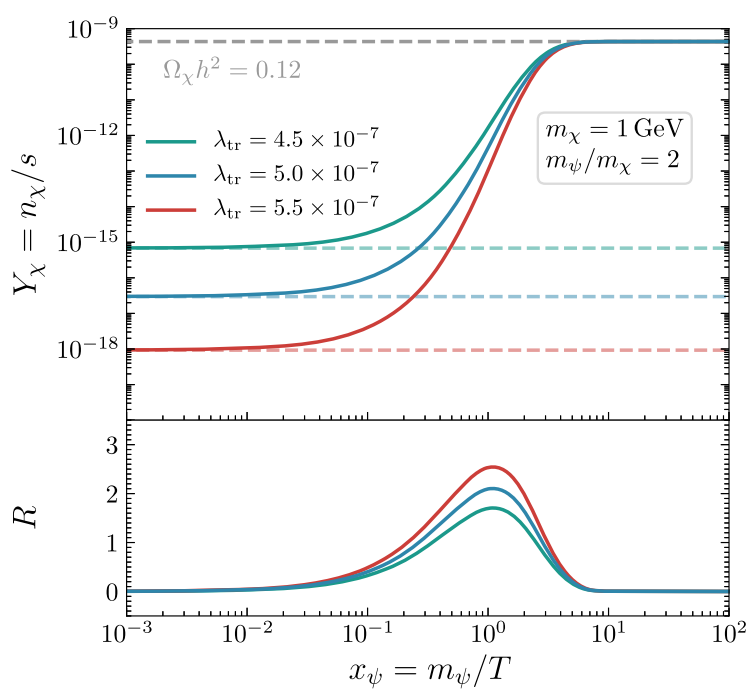

FIG. 2. Top: Number density of $\chi$ relative to the entropy density of the heat bath (solid lines) for $m_{\chi}=1 \mathrm{GeV}, m_{\psi}=2 \mathrm{GeV}$ and different values of the transformation coupling $\lambda_{\mathrm{tr}}$. For each value of $\lambda_{\mathrm{tr}}$, we fix the initial abundance of $\chi$ (dashed lines) such that the final abundance corresponds to the observed DM density. Bottom: $R$ value corresponding to the abundance evolution in the top panel.

dashed lines), the onset of exponential growth is clearly visible as $P$ becomes larger than $\sim 0.2$ for $x_{\psi} \gtrsim 0.01$ until it stalls because $P$ is heavily suppressed again for $x_{\psi} \gtrsim 5$. The figure also illustrates an attractive feature of exponential growth from a phenomenological point of view: the coupling strength required to match the observed DM relic abundance is only logarithmically sensitive to the initial abundance. In the examples shown here, e.g., decreasing the initial abundance by 4 orders of magnitude (from the green to the red line) is compensated by a mere increase of about $22 \%$ in $\lambda_{\text {tr }}$.

Exponential growth in nature.-It is intriguing how closely the evolution of the DM abundance in Fig. 2 mimics other well-known examples of exponential growth in nature-like, for example, the progression of an illness after an initial outbreak. In fact, we can formalize this analogy by referring to the SIR (susceptible, infected, and recovered) epidemiological model [35], where the number of infected individuals, $I$, evolves according to

$$
\dot{I}=\beta S I-\gamma I,
$$

with $S$ the number of susceptible individuals and $\beta$ and $\gamma$ the infection and recovery rates, respectively. We recognize that this is simply Eq. (1), in the limit $n_{\chi} \ll n_{\chi}^{\mathrm{eq}}$, after identifying $I \leftrightarrow n_{\chi}, S \leftrightarrow n_{\psi}^{\mathrm{eq}}, \beta \leftrightarrow\langle\sigma v\rangle_{\mathrm{tr}}$, and $\gamma \leftrightarrow 3 H$. This mathematically exact correspondence motivates us to further introduce 


$$
R \equiv \frac{\beta S}{\gamma}=\frac{n_{\psi}\langle\sigma v\rangle_{\mathrm{tr}}}{3 H}=\frac{\tilde{H}}{3 H} P
$$

where the final equality follows from Eq. (3). $R$ measures the number of transformation processes that each DM particle undergoes per Hubble time and is, through Eqs. (2) and (6), directly related to the final DM abundance.

Initial abundance.-In the above discussion, we have deliberately remained agnostic about the origin of $Y_{\chi}^{0}$ and simply treated this quantity as a free input parameter. We now outline various physical mechanisms that could generate such an initial DM abundance.

The first class of initial DM production mechanisms takes place much earlier than the typically rather short period where $\psi \psi \rightarrow \chi \psi$ processes dominate. This includes well-studied examples such as UV-dominated freeze-in $[36,37]$ or direct production from the decay of the inflaton or other heavy particles [38] but could also be related to more exotic examples like false vacua after a phase transition in the dark sector $[39,40]$ or by the evaporation of black holes. Common to all these scenarios is that the final DM abundance is independent of how exactly the initial abundance is set: the only phenomenologically relevant input is the DM abundance at the onset of the era of exponential growth, thus providing a direct map to the generic situation depicted in Fig. 2.

In the second class of relevant scenarios, the initial and exponential phases of DM production are intertwined. This is particularly relevant for IR dominated freeze-in rates [23] that are too small to explain the observed DM abundance without a subsequent phase of exponential growth. A nice feature of the mechanism proposed here is in fact that a nonvanishing freeze-in contribution due to the transformation coupling $\lambda_{\mathrm{tr}}$ is automatically built-in, as discussed below. In general, the Boltzmann equation including $2 \rightarrow 2$ freeze-in processes becomes

$$
\dot{n}_{\chi}+3 H n_{\chi} \simeq\langle\sigma v\rangle_{\mathrm{tr}} n_{\chi} n_{\psi}^{\mathrm{eq}}+\langle\sigma v\rangle_{\mathrm{fi}}\left(n_{\psi}^{\mathrm{eq}}\right)^{2},
$$

where $\langle\sigma v\rangle_{\mathrm{fi}}$ is the total cross section for $\psi \psi \rightarrow \chi \chi$ and $\psi \psi \rightarrow \chi \psi$. Since $n_{\psi}^{\text {eq }} \gg n_{\chi}$, a necessary condition for transformation processes to be non-negligible compared to traditional freeze-in is thus $\langle\sigma v\rangle_{\mathrm{fi}} \ll\langle\sigma v\rangle_{\mathrm{tr}}$. Once the two terms on the rhs of the above equation are of a similar size, on the other hand, $\psi \psi \rightarrow \chi \psi$ will very quickly take over due to the exponential growth of $n_{\chi}$.

We show the evolution of the DM abundance for this scenario in Fig. 3, assuming for simplicity that all amplitudes are constant. We also choose the same masses and transformation couplings as in Fig. 2 to facilitate comparison. Instead of fixing the initial abundance, however, we now fix the freeze-in coupling to result in the correct relic abundance (thus taking a vanishing DM abundance as the initial condition). The above-discussed three phasesfreeze-in, transformation, and the final flattening of the

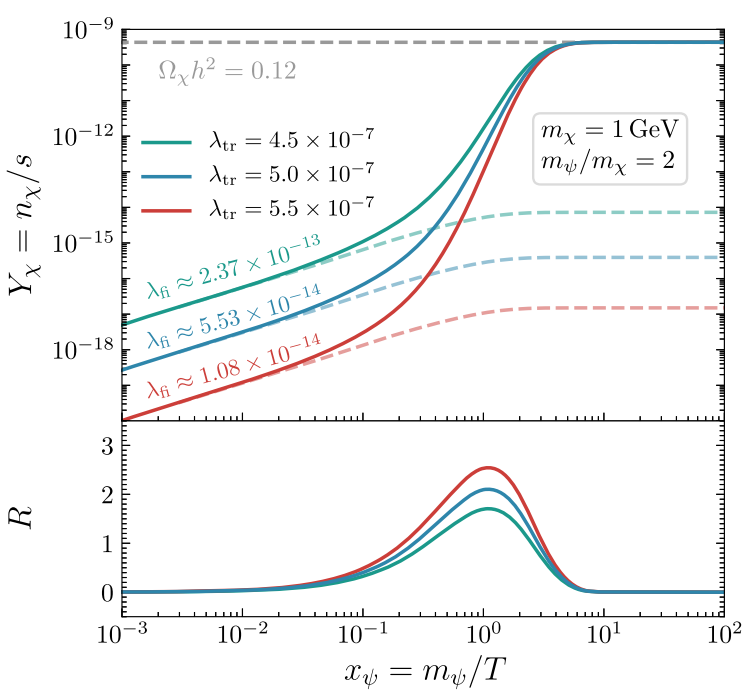

FIG. 3. As in Fig. 2 but now with a vanishing initial DM abundance and, on top of the transformation interaction, freeze-in production based on a constant matrix element. The coupling $\lambda_{\mathrm{fi}}$ for the latter is chosen such that the final abundance of $\chi$ corresponds to the observed DM density. Dashed lines show the would-be abundance from freeze-in alone (when setting $\lambda_{\mathrm{tr}}=0$, for which $\lambda_{\mathrm{fi}} \approx 5.81 \times 10^{-11}$ would give $\Omega_{\mathrm{DM}} h^{2}=0.12$ ).

abundance evolution curve-are clearly visible in the figure. We stress that this brings a new perspective to the widely studied freeze-in mechanism, which can trigger a subsequent phase of exponential growth in a rather natural way. It therefore becomes possible to satisfy the relic density constraint with significantly smaller couplings $\lambda_{\text {fi }}$ than generally assumed, opening up new parameter space where freeze-in is relevant for setting the DM energy density.

To further illustrate the last point, we show in Fig. 4 a full "phase diagram" of the combination of couplings $\lambda_{\text {tr }}$ and $\lambda_{\mathrm{fi}}$ that allow the production of DM from the heat bath for a fixed mass ratio of $m_{\psi} / m_{\chi}=1.2$. At each point in this plane, we thus adjust the mass $m_{\chi}$ such that $\Omega_{\mathrm{DM}} h^{2}=0.12$ (with dashed lines indicating isocontours of $m_{\chi}$ ). Depending on the couplings, the relic abundance can be set via different mechanisms. In the green (red) region, the relic is mainly set via freeze-in (freeze-out) of the process $\psi \psi \leftrightarrow \leftrightarrow \chi \chi$. In the blue (yellow) region, the relic density is instead mainly set via freeze-in (freeze-out) of the process $\psi \chi \leftrightarrow \chi \chi$. In regions where two colors overlap, more than one production mechanism can lead to the correct relic abundance, albeit for different masses. In the gray regions, either of the production mechanisms would require fully thermalized scalars with $m_{\psi}<0.5 \mathrm{MeV}$-for the mass ratio $m_{\psi} / m_{\chi}=1.2$ adopted for the purpose of this figure-which is in conflict with constraints from big bang nucleosynthesis (BBN) [41]. The blue region is bounded toward large values of $\lambda_{\text {tr }}$ since such couplings would-for exponential production-require masses above the unitarity limit of a thermal particle $m_{\psi}>140 \mathrm{TeV}$ [42]. 


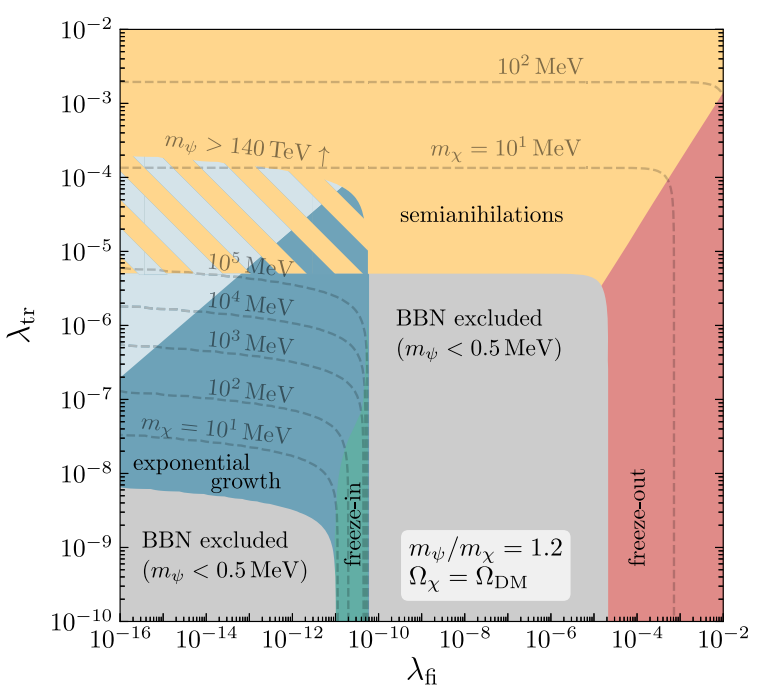

FIG. 4. Phase diagram of transformation $\left(\lambda_{\mathrm{tr}}\right)$ and freeze-in $\left(\lambda_{\mathrm{fi}}\right)$ couplings that can result in the correct DM abundance for a fixed mass ratio $m_{\psi} / m_{\chi}=1.2$. Colored regions indicate the respective mechanism that is responsible for thermal production, while dashed lines show the required value of $m_{\chi}$. Gray regions would require a new heat bath particle $\psi$ too light to be compatible with constraints from BBN [41]. In the light blue region an additional $2 \rightarrow 4$ freeze-in contribution is expected.

There is an irreducible $2 \rightarrow 4$ freeze-in contribution $\psi \psi \rightarrow 4 \chi$, with cross-section scaling as $\lambda_{\mathrm{tr}}^{4}$, which we estimate to dominate over $2 \rightarrow 2$ freeze-in within the light blue region. We neglect $2 \rightarrow 4$ processes in Fig. 4, which only have a logarithmic effect on the value of $\lambda_{\text {tr }}$ that results in the observed relic density. Finally, we note that $\lambda_{\text {tr }}$ generates $\lambda_{\mathrm{fi}}$ radiatively and, in the absence of fine-tuning, we expect $\lambda_{\mathrm{fi}} \gtrsim \lambda_{\mathrm{tr}}^{2} /(4 \pi)^{2}$. This bound is satisfied except in the light blue region where $2 \rightarrow 4$ processes are relevant.

Discussion.-We stress that exponential growth due to processes as depicted in Fig. 1 is by no means restricted to specific model realizations but is a general mechanism of DM production that essentially interpolates between the traditionally considered freeze-in and freeze-out regimes. At first glance it may seem worrisome that the dark matter density depends exponentially on the transformation cross section, implying that the cross section must be carefully chosen to match observation. But this can be turned around: in fact it implies that this mechanism is highly predictive, as manifested by the logarithmic sensitivity of the necessary cross section to the initial conditions. We also note that exponential sensitivity is quite common in nature and, for example, can result from renormalization group flows where IR parameters can be exponentially sensitive to UV parameters: the proton mass, e.g., depends exponentially on the size of the strong coupling at high energies [43].

It is worth pointing out that the mechanism proposed here works for a large range of different particle masses, not just the specific choice displayed in the examples above.
Limits from $\mathrm{BBN}$ or $\mathrm{CMB}$ on new light degrees of freedom $[41,44]$, as encountered in Fig. 4, could be significantly lowered by considering the possibility of $\psi$ being a SM particle. Larger values of $m_{\psi}$ would not affect the DM velocity dispersion at $T \sim m_{\chi}$ (but may sharpen modeldependent constraints from the decay of $\psi$, see below). Even a reverted mass hierarchy is possible, $m_{\chi}>m_{\psi}$, in which case it would be $m_{\chi}$ rather than $m_{\psi}$ that determines when the evolution of $n_{\chi} / s$ starts to flatten. For $m_{\chi}>m_{\psi}$, DM in general becomes unstable - but not necessarily on cosmological timescales. Such highly suppressed decays may potentially be visible in late-time observables related to cosmological structure formation or cosmic rays, making corresponding scenarios even more attractive.

Let us finish by considering the phenomenological consequences for a simple, concrete model realization, where $\psi$ is a real scalar and couples to the SM via a quartic Higgs portal coupling $\lambda_{h \psi}|H|^{2} \psi^{2} / 2$. The relic density of $\psi$ is then determined by standard freeze-out, exactly as for scalar singlet DM [45], and we fix $\lambda_{h \psi}$ by requiring $\Omega_{\psi}=0.1 \Omega_{\mathrm{DM}}$. This implies a unitarity limit of around $m_{\psi} \approx 44 \mathrm{TeV}$ [42] and a direct detection limit from Xenon1T [46] of $m_{\psi}>1 \mathrm{TeV}$ (obtained by rescaling the results from Ref. [45]); next-generation direct detection experiments will fully explore masses up to the unitarity limit [47]. In Fig. 5, we show these constraints in the plane $m_{\chi}-\lambda_{\mathrm{tr}}$. Here, we fix $\lambda_{\mathrm{fi}}$ such that the dominant DM

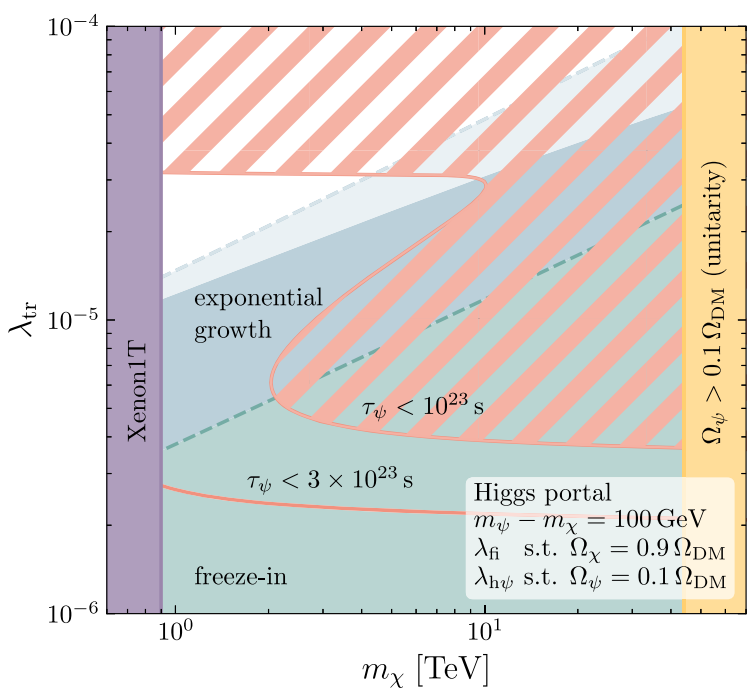

FIG. 5. Phenomenological consequences of one possible realization of exponential production, with $\psi$ coupling to the SM via the Higgs portal. For each value of $m_{\chi}$ and $\lambda_{\mathrm{tr}}$, the couplings $\lambda_{\mathrm{fi}}$ and $\lambda_{h \psi}$ are fixed such that $\psi(\chi)$ contributes $10 \%(90 \%)$ to the observed DM abundance. Green and blue areas correspond, as in Fig. 4, to different thermal productions regimes of $\chi$. The purple area is excluded by direct detection [46], while in the yellow area unitary constraints would imply an overproduction of $\psi$. Red lines (and hatched area) indicate where to naturally expect $\mathrm{CMB}$ signatures. Light blue shading as in Fig. 4. 
component satisfies $\Omega_{\chi}=0.9 \Omega_{\mathrm{DM}}$ and is mostly produced by transformation (blue) or freeze-in (green) processes (in the white area, $\Omega_{\chi}=0.9 \Omega_{\mathrm{DM}}$ may also be possible, but only via freeze-out or semiannihilations). Finally, the decay of $\psi$ to SM particles can impact the observed CMB power spectrum; for a $10 \% \mathrm{DM}$ subcomponent, this leads to a constraint of $\tau_{\psi} \gtrsim 10^{23} \mathrm{~s}$ [48], projected to tighten by a factor of $\sim 3$ with CMB-S4 [49]. Let us for simplicity adopt a fixed mass splitting of $m_{\psi}-m_{\chi}=100 \mathrm{GeV}$, such that only decays $\psi \rightarrow \chi b \bar{b}$ are relevant. These are dominated by either 2-loop or 3-loop diagrams, depending on the relative strength of transformation and freeze-in couplings. The naive size of these loops results in decay widths $\Gamma_{2} \sim$ $\mathcal{P} \lambda_{\text {tr }}^{2} \lambda_{\mathrm{fi}}^{2} \lambda_{h \psi}^{2} y_{b}^{2} /(4 \pi)^{8} / m_{\psi}$ and $\Gamma_{3} \sim \mathcal{P} \lambda_{\text {tr }}^{6} \lambda_{h \psi}^{2} y_{b}^{2} /(4 \pi)^{12} / m_{\psi}$, respectively, where $y_{b}$ is the bottom Yukawa coupling and $\mathcal{P} \simeq\left[m_{\psi}^{4}-m_{\chi}^{4}+4 m_{\psi}^{2} m_{\chi}^{2} \log \left(m_{\chi} / m_{\psi}\right)\right] /\left[512 \pi^{3} m_{\psi}^{2}\right]$ is the phase space in the limit $m_{\psi}-m_{\chi} \gg 2 m_{b}$. We use these expressions in Fig. 5 to indicate, with red lines, where to naturally expect CMB signatures in this model.

Conclusions. - We have introduced a novel type of DM production mechanism, where an initially tiny DM abundance is enhanced due to a process where DM particles convert bath particles into more DM particles. The DM abundance grows exponentially with time in stark contrast to models of freeze-in where the abundance grows only as a power law. Our mechanism complements both freeze-in and freeze-out thermal production scenarios in a generic way. Concrete model realizations lead, already in their simplest forms, to interesting phenomenological consequences. Further, and detailed, exploration of this new way of producing DM from the thermal bath thus appears highly warranted.

We thank Michael Geller for helpful conversations, including at a primordial stage. This work is supported by the Deutsche Forschungsgemeinschaft under Germany's Excellence Strategy_EXC 2121 "Quantum Universe"390833306, the F.R.S.-FNRS under the Excellence of Science (EoS) Project No. 30820817-be.h "The H boson gateway to physics beyond the Standard Model," and the National Science Foundation under Grant No. NSF PHY1748958. J. T. R. is further supported by the NSF CAREER Grant No. PHY-1554858, NSF Grant No. PHY-1915409, an award from the Alexander von Humboldt Foundation, and the European Research Council (ERC) under the EU Horizon 2020 Programme (ERC-CoG-2015-Proposal n. 682676 LDMThExp).

*Corresponding author.

torsten.bringmann@fys.uio.no

${ }^{\dagger}$ Corresponding author.

frederik.depta@desy.de

Corresponding author.

marco.hufnagel@ulb.ac.be
${ }^{\S}$ Corresponding author.

ruderman@nyu.edu

"Corresponding author.

kai.schmidt-hoberg@desy.de

[1] N. Aghanim et al. (Planck Collaboration), Planck 2018 results. VI. Cosmological parameters, Astron. Astrophys. 641, A6 (2020).

[2] B. W. Lee and S. Weinberg, Cosmological Lower Bound on Heavy Neutrino Masses, Phys. Rev. Lett. 39, 165 (1977).

[3] D. P. Finkbeiner and N. Weiner, Exciting dark matter and the INTEGRAL/SPI 511 keV signal, Phys. Rev. D 76, 083519 (2007).

[4] M. Pospelov, A. Ritz, and M. B. Voloshin, Secluded WIMP dark matter, Phys. Lett. B 662, 53 (2008).

[5] J. L. Feng, H. Tu, and H.-B. Yu, Thermal relics in hidden sectors, J. Cosmol. Astropart. Phys. 10 (2008) 043.

[6] M. Pospelov, Secluded $U(1)$ below the weak scale, Phys. Rev. D 80, 095002 (2009).

[7] K. Sigurdson, Hidden hot dark matter as cold dark matter, arXiv:0912.2346.

[8] C. Cheung, G. Elor, L. J. Hall, and P. Kumar, Origins of hidden sector dark matter I: Cosmology, J. High Energy Phys. 03 (2011) 042.

[9] T. Bringmann, P. F. Depta, M. Hufnagel, and K. SchmidtHoberg, Precise dark matter relic abundance in decoupled sectors, Phys. Lett. B 817, 136341 (2021).

[10] R. T. D'Agnolo and J. T. Ruderman, Light Dark Matter from Forbidden Channels, Phys. Rev. Lett. 115, 061301 (2015).

[11] R. T. D’Agnolo, D. Liu, J. T. Ruderman, and P.-J. Wang, Forbidden dark matter annihilations into Standard Model particles, J. High Energy Phys. 06 (2021) 103.

[12] D. Pappadopulo, J. T. Ruderman, and G. Trevisan, Dark matter freeze-out in a nonrelativistic sector, Phys. Rev. D 94, 035005 (2016).

[13] M. Farina, D. Pappadopulo, J. T. Ruderman, and G. Trevisan, Phases of cannibal dark matter, J. High Energy Phys. 12 (2016) 039.

[14] R. T. D'Agnolo, D. Pappadopulo, and J. T. Ruderman, Fourth Exception in the Calculation of Relic Abundances, Phys. Rev. Lett. 119, 061102 (2017).

[15] M. Garny, J. Heisig, B. Lülf, and S. Vogl, Coannihilation without chemical equilibrium, Phys. Rev. D 96, 103521 (2017).

[16] R. T. D’Agnolo, D. Pappadopulo, J. T. Ruderman, and P.-J. Wang, Thermal Relic Targets with Exponentially Small Couplings, Phys. Rev. Lett. 124, 151801 (2020).

[17] E. D. Kramer, E. Kuflik, N. Levi, N. J. Outmezguine, and J. T. Ruderman, Heavy Thermal Dark Matter from a New Collision Mechanism, Phys. Rev. Lett. 126, 081802 (2021).

[18] E. Kuflik, M. Perelstein, NicolasRey-Le. Lorier, and Y.-D. Tsai, Elastically Decoupling Dark Matter, Phys. Rev. Lett. 116, 221302 (2016).

[19] P. J. Fitzpatrick, H. Liu, T. R. Slatyer, and Y.-D. Tsai, New pathways to the relic abundance of vector-portal dark matter, arXiv:2011.01240.

[20] E. D. Carlson, M. E. Machacek, and L. J. Hall, Self-interacting dark matter, Astrophys. J. 398, 43 (1992).

[21] Y. Hochberg, E. Kuflik, T. Volansky, and J. G. Wacker, Mechanism for Thermal Relic Dark Matter of Strongly 
Interacting Massive Particles, Phys. Rev. Lett. 113, 171301 (2014).

[22] J. Smirnov and J. F. Beacom, New Freezeout Mechanism for Strongly Interacting Dark Matter, Phys. Rev. Lett. 125, 131301 (2020).

[23] L. J. Hall, K. Jedamzik, J. March-Russell, and S. M. West, Freeze-in production of FIMP dark matter, J. High Energy Phys. 03 (2010) 080.

[24] X. Chu, T. Hambye, and M. H. G. Tytgat, The four basic ways of creating dark matter through a portal, J. Cosmol. Astropart. Phys. 05 (2012) 034.

[25] X. Chu, Y. Mambrini, J. Quevillon, and B. Zaldivar, Thermal and non-thermal production of dark matter via Z'-portal(s), J. Cosmol. Astropart. Phys. 01 (2014) 034.

[26] A. Falkowski, E. Kuflik, N. Levi, and T. Volansky, Light dark matter from leptogenesis, Phys. Rev. D 99, 015022 (2019), arXiv:1712.07652 [hep-ph].

[27] H. An, R. Huo, and W. Liu, KeV scale frozen-in selfinteracting fermionic dark matter, arXiv:1812.05699.

[28] T. Hambye, M. H. G. Tytgat, J. Vandecasteele, and L. Vanderheyden, Dark matter from dark photons: A taxonomy of dark matter production, Phys. Rev. D 100, 095018 (2019).

[29] C. Mondino, M. Pospelov, J. T. Ruderman, and O. Slone, Dark Higgs dark matter, Phys. Rev. D 103, 035027 (2021).

[30] G. Bélanger, C. Delaunay, A. Pukhov, and B. Zaldivar, Dark matter abundance from the sequential freeze-in mechanism, Phys. Rev. D 102, 035017 (2020).

[31] N. Bernal, Boosting freeze-in through thermalization, J. Cosmol. Astropart. Phys. 10 (2020) 006.

[32] J. March-Russell, H. Tillim, and S. M. West, Reproductive freeze-in of self-interacting dark matter, Phys. Rev. D 102, 083018 (2020).

[33] A. Berlin, WIMPs with GUTs: Dark Matter Coannihilation with a Lighter Species, Phys. Rev. Lett. 119, 121801 (2017).

[34] J. Edsjö and P. Gondolo, Neutralino relic density including coannihilations, Phys. Rev. D 56, 1879 (1997).

[35] W. O. Kermack and A. G. McKendrick, A contribution to the mathematical theory of epidemics, Proc. R. Soc. A 115, 700 (1927).
[36] T. Moroi, H. Murayama, and M. Yamaguchi, Cosmological constraints on the light stable gravitino, Phys. Lett. B 303, 289 (1993).

[37] M. Bolz, A. Brandenburg, and W. Buchmuller, Thermal production of gravitinos, Nucl. Phys. B606, 518 (2001); Erratum, Nucl. Phys. B790, 336(E) (2008).

[38] F. Takahashi, Gravitino dark matter from inflaton decay, Phys. Lett. B 660, 100 (2008).

[39] E. Witten, Cosmic separation of phases, Phys. Rev. D 30, 272 (1984).

[40] P. Asadi, E. D. Kramer, E. Kuflik, G. W. Ridgway, T. R. Slatyer, and J. Smirnov, Thermal squeezeout of dark matter, arXiv:2103.09827.

[41] P. F. Depta, M. Hufnagel, K. Schmidt-Hoberg, and S. Wild, BBN constraints on the annihilation of $\mathrm{MeV}$-scale dark matter, J. Cosmol. Astropart. Phys. 04 (2019) 029.

[42] J. Smirnov and J. F. Beacom, TeV-Scale thermal WIMPs: Unitarity and its consequences, Phys. Rev. D 100, 043029 (2019).

[43] G. W. Anderson and D. J. Castano, Measures of fine tuning, Phys. Lett. B 347, 300 (1995).

[44] N. Sabti, J. Alvey, M. Escudero, M. Fairbairn, and D. Blas, Refined bounds on MeV-scale thermal dark sectors from BBN and the CMB, J. Cosmol. Astropart. Phys. 01 (2020) 004.

[45] J. M. Cline, P. Scott, K. Kainulainen, and C. Weniger, Update on scalar singlet dark matter, Phys. Rev. D 88, 055025 (2013); Erratum, Phys. Rev. D 92, 039906 (2015).

[46] E. Aprile et al. (XENON Collaboration), Dark Matter Search Results from a One Ton-Year Exposure of XENON1T, Phys. Rev. Lett. 121, 111302 (2018).

[47] E. Aprile et al. (XENON Collaboration), Projected WIMP sensitivity of the XENONnT dark matter experiment, J. Cosmol. Astropart. Phys. 11 (2020) 031.

[48] T. R. Slatyer and C.-L. Wu, General constraints on dark matter decay from the cosmic microwave background, Phys. Rev. D 95, 023010 (2017).

[49] K. N. Abazajian et al. (CMB-S4 Collaboration), CMB-S4 Science Book, First Edition, arXiv:1610.02743. 